\title{
A Numerical Study of Multiple Solutions for Laminar Flows in a Porous and Moving Channel
}

\author{
Fen Wang ${ }^{1}$, Ping Lin ${ }^{1,2, *}$, Lin $\mathrm{Li}^{1}$ and Yongyue Jiang ${ }^{3}$ \\ ${ }^{1}$ Beijing Key Laboratory for Magneto-Photoelectrical Composite and Interface \\ Science, School of Mathematics and Physics, University of Science and Technology \\ Beijing, Beijing 100083, China \\ ${ }^{2}$ Division of Mathematics, University of Dundee, Dundee DD1 4HN, United \\ Kingdom \\ ${ }^{3}$ Beijing Time Technologies Co., Ltd., Kaituo Road 17, Beijing, China \\ Received 8 January 2016; Accepted (in revised version) 14 February 2017
}

\begin{abstract}
In this paper, based on the finite element formulation, we focus on multiple solutions and their evolution with time for a laminar flow in a permeable channel with expanding or contracting walls. Both Newtonian fluid and micropolar fluid are considered. For the Newtonian fluid model, we find that the profile of the unique solution in the case of injection remains the same for long time, which indicates that the solution may be stable. On the other hand, in the case of large suction, the profile of multiple solutions changes in time, which suggests that the multiple solutions may be unstable. Similar behaviors and conclusions are observed for the micropolar fluid model under different boundary parameters.
\end{abstract}

AMS subject classifications: 34A99, 35A22, 35K15

Key words: Newtonian fluid, micropolar fluid, multiple solutions, finite element method, bvp4c.

\section{Introduction}

Studies of laminar flows in a porous channel have received considerable attention in recent years due to their wide range of applications in a number of engineering and biological models, for examples, the transport of biological fluids through vessels, the modeling of blood and air circulation in a respiratory system.

In the study of the refrigeration of steam and the separation process of $U_{235}$ (a radioactive isotope of uranium element whose neutron number is 143), or in a gas diffusion process, Berman [1] obtained an asymptotic solution for small Reynolds numbers using a regular perturbation method assuming a steady, incompressible and laminar flow through a two dimensional porous channel with stationary walls in 1953. In the study, Berman also assumed that normal velocity is independent of the stream-wise coordinate and a fully developed flow. One of the key ideas in the proof is to transform the Navier-Stokes equations

${ }^{*}$ Corresponding author. Email address: plin@maths.dundee.ac.uk (P. Lin) 
to a system of nonlinear ordinary differential equations that contains only a permeation Reynolds number by introducing the stream function.

In the following decades, many researchers have extended and generalized Berman's work, particularly in the following three directions.

First of all, numerous studies of channel flows through porous and rigid walls have been conducted. For both small and large Reynolds numbers Re, for example, Terrill [2] obtained the solutions using a perturbation method. Using an integral equations approach, Proudman [3] also investigated the solution for large Reynolds number cases. Later on, again using a singular perturbation method, Yuan [4] obtained solutions for the case of a large injection rate. Using the average method, Morduchow [5] obtained an analytical solution in the entire injection range.

Secondly, in seeking further generalization, Dauenhauer and Majdalani [6] considered the case of a laminar flow in a porous channel with expanding or contracting walls in 2003. By introducing an expansion ratio $\alpha$ and assuming it is a constant, the authors reduced the governing equations to an ordinary differential equation only involving the Reynolds number and the expansion ratio. The Runge-Kutta method coupled with a shooting method is used to obtained asymptotic solutions over a modest range of $R e$ and $\alpha$. Furthermore, not only the authors carried on the mechanism analysis to the related physical quantities of their model, but also obtained the relations between $R e$ and $\alpha$.

Thirdly, the existence of multiple solution is revealed in the course of discussions on Berman's work. Raithby [7] first discovered two numerical solutions in the research of the heat transfer in a rectangular channel at the entrance. In 1976, Robinson [8] carried out a numerical research about the multiplicity of the solutions for the flow in a porous channel and drawn the following conclusions. When $R e<12.165$, the corresponding ordinary differential equation by the similarity transformation has only one numerical solution; but when $12.165 \leq R e<\infty$, the ODE has three solutions, which are labeled type $I$, type II, type III. Meanwhile, Raithby [7] also obtained two asymptotic solutions through a singular perturbation method. As for the stability analysis on a laminar flow, Sobey and Drazin [9] were the pioneers to investigate the stability and bifurcation of a symmetric flow in a two-dimensional rectangular regions in 1986. According to Sobey and Drazin, there is a unique solution which is stable for small Reynolds numbers. The unique solution will become unstable as the Reynolds reaches a critical value. Since then, many other interesting studies on this subject have been appeared in the literature, for examples, by Zaturska et al. [10], Waton et al. [11], Durlofsky and Brady [12], Cox and King [13].

Recently, using a homotopy method, Xu et al. [14] investigated multiple solutions of the Navier-Stokes equations for the Newtonian fluid model in a permeable channel with orthogonally moving walls in the context of a suction process assuming constant Reynolds number and the expansion ratio $\alpha$. Two or three solutions are obtained under some values of $R e$ and $\alpha$. The authors also considered a more general case that $\alpha$ is a function of time and concluded that the solution reaches the steady state faster in the case of a larger suction rate $\alpha(t)$ compared that with a constant $\alpha$. Following Xu et al. [14]'s work, Si et al. [15] investigated the same problem and obtained dual solutions for large suction rate by using a singular perturbation method. Based on a finite element method, Xu et al. [16] 


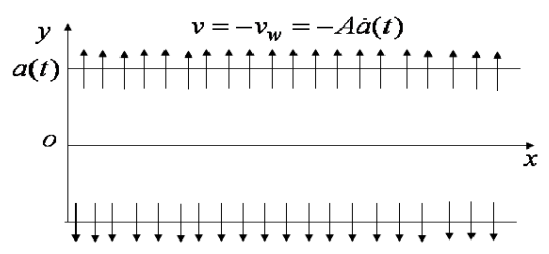

Figure 1: A diagram of the set-up the problem, a porous domain and a moving channel.

further examined the problem described in [14] and obtained two or three numerical solutions that agreed well with the corresponding analytic approximation solutions in [14] with large suction rates. However, to our best knowledge, finite element methods are rarely used to study the multiple solutions of the laminar flow in a channel. Moreover, few research can be found in the literature about the time evolution of the multiple solutions.

Note that, many studies in the past are only for Newtonian fluids. However, in many important applications, non-Newtonian fluids are equally or more important. For example, quite a number of papers pointed out that the blood flow in a vessel can be simulated by a micropolar fluid flow model which is non-Newtonian. The theory of micropolar fluids was first established by Eringen $[17,18]$. Intensive studies on the micropolar fluid flow in a channel have been carried out by Darvishi et al. [19], Ashraf et al. [20], Rashidi et al. [21], Ziabakhsh and Domairry [22], Joneidi et al. [23] and Si et al. [24]. Nevertheless, so far, few literature deal with multiple solutions of a micropolar fluid in a porous channel with accelerating walls.

Inspired by all the above-mentioned research, in this paper, we will study multiple solutions and their spatial variations with time for laminar flows in a porous and moving channel using a finite element method. We solve the full Navier-Stokes equations and thus we have removed different restrictions in previous research using perturbation and asymptotic expansions and changing PDEs to ODEs etc. Note that finite element methods have been widely applied to solve the Navier-Stokes equations, see, example [25-27]. In this paper we aim at examining the stability of multiple solutions by solving the unsteady Navier-Stokes equations.

The rest of paper is organized as follows. In Section 2, we introduce two fluids flow models in a porous channel with expanding or contracting walls, one is a Newton fluid flow model, and the other is a micropolar fluid flow model. In Section 3, we derive the variational form of the governing equations using the integration by parts and the Green theorem. In Section 4, we will describe the fully discretized finite element scheme for the two models. In Section 5, we present our numerical solutions and compare our results with those in the literature.

\section{Two models}

\subsection{Newtonian fluid flow model}

As illustrated in Fig. 1, we assume that a rectangular channel is porous and expanding 
or contracting in the transverse direction simultaneously. Furthermore, the fluid is injected or sucked uniformly at an absolute velocity $v_{w}$ through the opposing two walls. We assume that the height of the channel is $2 a(t)$, and its length is semi-infinite. The flow velocity components are defined as $u, v$ in the $x$-direction and $y$-direction, respectively. At $x=0$, the velocity $u, v$ are assumed to be zero. The Navier-Stokes equations (see [6]) of the fluid flow model are,

$$
\begin{aligned}
& \frac{\partial u}{\partial x}+\frac{\partial v}{\partial y}=0, \\
& \frac{\partial u}{\partial t}+u \frac{\partial u}{\partial x}+v \frac{\partial u}{\partial y}=-\frac{1}{\rho} \frac{\partial p}{\partial x}+v\left(\frac{\partial^{2} u}{\partial x^{2}}+\frac{\partial^{2} u}{\partial y^{2}}\right), \\
& \frac{\partial v}{\partial t}+u \frac{\partial v}{\partial x}+v \frac{\partial v}{\partial y}=-\frac{1}{\rho} \frac{\partial p}{\partial y}+v\left(\frac{\partial^{2} v}{\partial x^{2}}+\frac{\partial^{2} v}{\partial y^{2}}\right),
\end{aligned}
$$

where $\rho, v, t$ are the density, kinematic viscosity and time, respectively. The crossflow Reynolds number is defined as $R e=a v_{w} / v$, where $R e>0$ is for injection and $R e<0$ is for suction. The corresponding boundary conditions are,

$$
\begin{array}{lll}
u=0, & v=-v_{w} ; & y=a(t), \\
u=0, & v=v_{w} ; & y=-a(t), \\
u=0, & v=0 ; & x=0 .
\end{array}
$$

We nondimensionalize the governing equations and boundary conditions by introducing the following dimensionless variables,

$$
\begin{aligned}
& t^{*}=\frac{v_{w}}{a(t)} t, \quad x^{*}=\frac{x}{a(t)}, \quad y^{*}=\frac{y}{a(t)}, \\
& u^{*}=\frac{u}{v_{w}}, \quad v^{*}=\frac{v}{v_{w}}, \quad p^{*}=\frac{a p}{\rho v_{w} v} \text {. }
\end{aligned}
$$

The original Navier-Stokes equations become

$$
\begin{aligned}
& \frac{\partial u}{\partial t}=\left(\frac{v_{w}^{2}}{a}-\frac{v_{w} t^{*}}{a} \dot{a}\right) \frac{\partial u^{*}}{\partial t^{*}}-\frac{v_{w}}{a} \dot{a} x^{*} \frac{\partial u^{*}}{\partial x^{*}}-\frac{v_{w}}{a} \dot{a} y^{*} \frac{\partial u^{*}}{\partial y^{*}}, \\
& \frac{\partial v}{\partial t}=\left(\frac{v_{w}^{2}}{a}-\frac{v_{w} t^{*}}{a} \dot{a}\right) \frac{\partial v^{*}}{\partial t^{*}}-\frac{v_{w}}{a} \dot{a} x^{*} \frac{\partial v^{*}}{\partial x^{*}}-\frac{v_{w}}{a} \dot{a} y^{*} \frac{\partial v^{*}}{\partial y^{*}}, \\
& \frac{\partial u}{\partial x}=\frac{v_{w}}{a} \frac{\partial u^{*}}{\partial x^{*}}, \quad \frac{\partial u}{\partial y}=\frac{v_{w}}{a} \frac{\partial u^{*}}{\partial y^{*}}, \quad \frac{\partial^{2} u}{\partial x^{2}}=\frac{v_{w}}{a^{2}} \frac{\partial^{2} u^{*}}{\partial x^{* 2}}, \quad \frac{\partial^{2} u}{\partial y^{2}}=\frac{v_{w}}{a^{2}} \frac{\partial^{2} u^{*}}{\partial y^{* 2}}, \\
& \frac{\partial v}{\partial x}=\frac{v_{w}}{a} \frac{\partial v^{*}}{\partial x^{*}}, \quad \frac{\partial v}{\partial y}=\frac{v_{w}}{a} \frac{\partial v^{*}}{\partial y^{*}}, \quad \frac{\partial^{2} v}{\partial x^{2}}=\frac{v_{w}}{a^{2}} \frac{\partial^{2} v^{*}}{\partial^{2} x^{* 2}}, \quad \frac{\partial^{2} v}{\partial y^{2}}=\frac{v_{w}}{a^{2}} \frac{\partial^{2} v^{*}}{\partial y^{* 2}} \text {. }
\end{aligned}
$$

Thus, Eqs. (2.1a)-(2.1c) and the corresponding boundary conditions (2.2a)-(2.2c) are changed to the following, 


$$
\begin{aligned}
& \frac{\partial u^{*}}{\partial x^{*}}+\frac{\partial v^{*}}{\partial y^{*}}=0, \\
& \left(R e-\alpha t^{*}\right) \frac{\partial u^{*}}{\partial t^{*}}-\alpha x \frac{\partial u^{*}}{\partial x^{*}}-\alpha y \frac{\partial u^{*}}{\partial y^{*}}+\operatorname{Re}\left(u^{*} \frac{\partial u^{*}}{\partial x^{*}}+v^{*} \frac{\partial u^{*}}{\partial y^{*}}\right) \\
= & -\frac{\partial p^{*}}{\partial x^{*}}+\left(\frac{\partial^{2} u^{*}}{\partial x^{* 2}}+\frac{\partial^{2} u^{*}}{\partial y^{* 2}}\right), \\
& \left(R e-\alpha t^{*}\right) \frac{\partial v^{*}}{\partial t^{*}}-\alpha x \frac{\partial v^{*}}{\partial x^{*}}-\alpha y \frac{\partial u^{*}}{\partial y^{*}}+R e\left(u^{*} \frac{\partial v^{*}}{\partial x^{*}}+v^{*} \frac{\partial v^{*}}{\partial y^{*}}\right) \\
= & -\frac{\partial p^{*}}{\partial x^{*}}+\left(\frac{\partial^{2} v^{*}}{\partial x^{* 2}}+\frac{\partial^{2} v^{*}}{\partial y^{* 2}}\right) .
\end{aligned}
$$

Without loss of generality, removing the asterisk, we have the equations and the boundary conditions,

$$
\begin{aligned}
& \frac{\partial u}{\partial x}+\frac{\partial v}{\partial y}=0, \\
& (R e-\alpha t) \frac{\partial u}{\partial t}-\alpha x \frac{\partial u}{\partial x}-\alpha y \frac{\partial u}{\partial y}+R e\left(u \frac{\partial u}{\partial x}+v \frac{\partial u}{\partial y}\right)=-\frac{\partial p}{\partial x}+\left(\frac{\partial^{2} u}{\partial x^{2}}+\frac{\partial^{2} u}{\partial y^{2}}\right) \\
& (R e-\alpha t) \frac{\partial v}{\partial t}-\alpha x \frac{\partial v}{\partial x}-\alpha y \frac{\partial u}{\partial y}+\operatorname{Re}\left(u \frac{\partial v}{\partial x}+v \frac{\partial v}{\partial y}\right)=-\frac{\partial p}{\partial x}+\left(\frac{\partial^{2} v}{\partial x^{2}}+\frac{\partial^{2} v}{\partial y^{2}}\right), \\
& \begin{array}{lll}
u=0, & v=-1, & y=1, \\
u=0, & v=1, & y=-1 \\
u=0, & v=0, & x=0 .
\end{array}
\end{aligned}
$$

\subsection{Micropolar fluid flow model}

Now we consider the micro-polar flow fluid in a channel as illustrated in Fig. 1. The stream-wise and normal velocity components are defined as $u, v$ and $N$ is the microrotation, respectively. We ignore the effects of body forces and their coupling. Then the governing equations (see [24]) of the micropolar fluid flow in a channel are the following,

$$
\begin{aligned}
& \frac{\partial u}{\partial x}+\frac{\partial v}{\partial y}=0 \\
& \frac{\partial u}{\partial t}+u \frac{\partial u}{\partial x}+v \frac{\partial u}{\partial y}=-\frac{1}{\rho} \frac{\partial p}{\partial x}+\left(v+\frac{\kappa}{\rho}\right)\left(\frac{\partial^{2} u}{\partial x^{2}}+\frac{\partial^{2} u}{\partial y^{2}}\right)+\frac{\kappa}{\rho} \frac{\partial N}{\partial y} \\
& \frac{\partial v}{\partial t}+u \frac{\partial v}{\partial x}+v \frac{\partial v}{\partial y}=-\frac{1}{\rho} \frac{\partial p}{\partial y}+\left(v+\frac{\kappa}{\rho}\right)\left(\frac{\partial^{2} v}{\partial x^{2}}+\frac{\partial^{2} v}{\partial y^{2}}\right)-\frac{\kappa}{\rho} \frac{\partial N}{\partial x} \\
& \rho j\left(\frac{\partial N}{\partial t}+u \frac{\partial N}{\partial x}+v \frac{\partial N}{\partial y}\right)=-\kappa\left(2 N+\frac{\partial u}{\partial y}-\frac{\partial v}{\partial x}\right)+\gamma\left(\frac{\partial^{2} N}{\partial x^{2}}+\frac{\partial^{2} N}{\partial y^{2}}\right),
\end{aligned}
$$


where $\rho, v, \kappa, j, \gamma, t$ are the density, kinematic viscosity, micro-rotation parameter, microrotation density, spin-gradient velocity, time, respectively, just as in [24], and $\gamma$ is assumed to be

$$
\gamma=\left(\mu+\frac{\kappa}{2}\right) j,
$$

in which $\mu$ is the dynamic viscosity. The corresponding boundary conditions are

$$
\begin{array}{llll}
u=0, & v=v_{w}, & N=-s \frac{\partial u}{\partial y} ; & y=a(t), \\
u=0, & v=-v_{w}, & N=-s \frac{\partial u}{\partial y} ; & y=-a(t), \\
u=0, & v=0, & N=0, & x=0,
\end{array}
$$

in which $s$ is a boundary parameter that is used to model the extent to which microelements are free to rotate in the vicinity of the channel walls, and there is a concrete description for different values of $s$ in [24].

We introduce the dimensionless variables as (2.3), and for the micro-rotation $N$, it is defined as $N^{*}=a(t) N / v_{w}$, thus we get,

$$
\begin{aligned}
& \frac{\partial N}{\partial t}=-\frac{v_{w}}{a} \dot{a} N^{*}-\left(\frac{v_{w}^{2}}{a}-\frac{v_{w}}{a} \dot{a}\right) \frac{\partial N^{*}}{\partial t^{*}}-\frac{v_{w}}{a} \dot{a} x^{*} \frac{\partial N^{*}}{\partial x^{*}}-\frac{v_{w}}{a} \dot{a} y^{*} \frac{\partial N^{*}}{\partial y^{*}}, \\
& \frac{\partial N}{\partial x}=\frac{v_{w}}{a^{2}} \frac{\partial N^{*}}{\partial x^{*}}, \quad \frac{\partial N}{\partial y}=\frac{v_{w}}{a^{2}} \frac{\partial N^{*}}{\partial y^{*}}, \quad \frac{\partial^{2} N}{\partial x^{2}}=\frac{v_{w}}{a^{3}} \frac{\partial^{2} N^{*}}{\partial x^{* 2}}, \quad \frac{\partial^{2} N}{\partial y^{2}}=\frac{v_{w}}{a^{3}} \frac{\partial^{2} N^{*}}{\partial y^{* 2}} .
\end{aligned}
$$

Combing with (2.4a)-(2.4d) and removing the asterisk, Eqs. (2.7a)-(2.7d) are finally transferred to,

$$
\begin{aligned}
& \frac{\partial u}{\partial x}+\frac{\partial v}{\partial y}=0, \\
& (R e-\alpha t) \frac{\partial u}{\partial t}-\alpha x \frac{\partial u}{\partial x}-\alpha y \frac{\partial u}{\partial y}+\operatorname{Re}\left(u \frac{\partial u}{\partial x}+v \frac{\partial u}{\partial y}\right) \\
= & -\frac{\partial p}{\partial x}+(1+K)\left(\frac{\partial^{2} u}{\partial x^{2}}+\frac{\partial^{2} u}{\partial y^{2}}\right)+K \frac{\partial N}{\partial y}, \\
& (\operatorname{Re}-\alpha t) \frac{\partial v}{\partial t}-\alpha x \frac{\partial v}{\partial x}-\alpha y \frac{\partial u}{\partial y}+\operatorname{Re}\left(u \frac{\partial v}{\partial x}+v \frac{\partial v}{\partial y}\right) \\
= & -\frac{\partial p}{\partial x}+(1+K)\left(\frac{\partial^{2} v}{\partial x^{2}}+\frac{\partial^{2} v}{\partial y^{2}}\right)-K \frac{\partial N}{\partial x}, \\
& (\operatorname{Re}-\alpha t) \frac{\partial N}{\partial t}-\alpha N-\alpha x \frac{\partial v}{\partial x}-\alpha y \frac{\partial u}{\partial y}+\operatorname{Re}\left(u \frac{\partial N}{\partial x}+v \frac{\partial N}{\partial y}\right) \\
= & -K\left(2 N+\frac{\partial u}{\partial y}-\frac{\partial v}{\partial x}\right)+\left(1+\frac{K}{2}\right)\left(\frac{\partial^{2} N}{\partial x^{2}}+\frac{\partial^{2} N}{\partial y^{2}}\right) .
\end{aligned}
$$


These are coupled with the following boundary conditions,

$$
\begin{array}{llll}
u=0, & v=1, & N=-s \frac{\partial u}{\partial y}, & y=1, \\
u=0, & v=-1, & N=-s \frac{\partial u}{\partial y}, & y=-1, \\
u=0, & v=0, & N=0, & x=0 .
\end{array}
$$

Since the solution domain in which finite element method is adopted has to be closed, we need to choose a closed region of the channel and give four corresponding boundary conditions at four boundaries of the domain. While boundary conditions of the channel are already known along the left, bottom, and upper boundaries, which are shown in (2.2a)(2.2c) for the first model and (2.9a)-(2.9c) for the micropolar fluid model, we just need to construct a boundary condition along the right side of the closed domain.

For the Newtonian fluid model, we propose new conditions of $u, v$ at the right side as $\frac{\partial u}{\partial x}+\frac{\partial v}{\partial y}=0, \frac{\partial v}{\partial n}=0$, respectively, where $n$ is the outward normal direction of the domain, motivated by the mass conservation law and the outlet conditions $\frac{\partial u}{\partial n}=0, \frac{\partial v}{\partial n}=0$ proposed in [16]. Then we obtain the dimensionless conditions

$$
\frac{\partial u}{\partial x}+\frac{\partial v}{\partial y}=0, \quad \frac{\partial v}{\partial n}=0
$$

through (2.3) and removing the asterisk.

Similarly, for micropolar fluid model, we can construct the dimensionless boundary conditions of $u, v, N$ at the right side as

$$
\frac{\partial u}{\partial x}+\frac{\partial v}{\partial y}=0, \quad \frac{\partial v}{\partial n}=0, \quad N-\frac{\partial N}{\partial n}=0
$$

\section{Variational formulation}

Let $\Omega$ be the domain of the channel, $\Gamma$ be the boundary which consists of $\Gamma_{1}, \Gamma_{2}, \Gamma_{3}, \Gamma_{4}$, representing the bottom, right, upper, left, of the rectangular channel. We assume that $\Gamma$ is smooth. All unknown variables should be in appropriate Sobolev space. We consider $u, v$ in $H^{1}(\Omega)$ and $p$ in $L^{2}(\Omega)$, thus a weak formulation or direct variational of Eqs. (2.6a)-(2.6c) may be drived by multiplying with $q$ in $L^{2}(\Omega), w_{1}, w_{2}$ in $H^{1}(\Omega)$, respectively, then using integration by parts and the Green theorem.

As in Hua et al. [28] and Shi et al. [29], we use the penalty formulation to deal with the divergence-free conditon (2.6a), which not only avoids applying an artificial boundary condition of the pressure, but also improves the stability of the problem. The penalty form is

$$
\iint_{\Omega}\left(\frac{\partial u}{\partial x}+\frac{\partial v}{\partial y}+\varepsilon p\right) q d x d y=0,
$$


where $\varepsilon$ is a small penalty, which is $\varepsilon=10^{-7}$ in our computation. Note that the larger the $\varepsilon$ is, the more stable the problem will be.

According to the discussions above, we have the weak form of the Eqs. (2.6a)-(2.6c) as follows,

$$
\begin{aligned}
& \iint_{\Omega}\left(\frac{\partial u}{\partial x}+\frac{\partial v}{\partial y}+\varepsilon p\right) q d x d y=0 \\
& \iint_{\Omega}\left[(\operatorname{Re}-\alpha t) \frac{\partial u}{\partial t} w_{1}-\alpha x \frac{\partial u}{\partial x} w_{1}-\alpha y \frac{\partial u}{\partial y} w_{1}+\operatorname{Re}\left(u \frac{\partial u}{\partial x} w_{1}+v \frac{\partial u}{\partial y} w_{1}\right)-p \frac{\partial w_{1}}{\partial x}\right. \\
& \left.\quad+\left(\frac{\partial u}{\partial x} \frac{\partial w_{1}}{\partial x}+\frac{\partial u}{\partial y} \frac{\partial w_{1}}{\partial y}\right)\right] d x d y+\int_{\Gamma_{2}} p w_{1} d s+\int_{\Gamma_{2}} w_{1} \frac{\partial v}{\partial y} d s=0 \\
& \iint_{\Omega}\left[(\operatorname{Re}-\alpha t) \frac{\partial v}{\partial t} w_{2}-\alpha x \frac{\partial v}{\partial x} w_{2}-\alpha y \frac{\partial v}{\partial y} w_{2}+\operatorname{Re}\left(u \frac{\partial v}{\partial x} w_{2}+v \frac{\partial v}{\partial y} w_{2}\right)-p \frac{\partial w_{2}}{\partial y}\right. \\
& \left.\quad+\left(\frac{\partial v}{\partial x} \frac{\partial w_{2}}{\partial x}+\frac{\partial v}{\partial y} \frac{\partial w_{2}}{\partial y}\right)\right] d x d y=0
\end{aligned}
$$

For micropolar fluid model, using the same procedure as for the Newtonian fluid model, we can get the variational form of (2.11a)-(2.11d),

$$
\begin{gathered}
\iint_{\Omega}\left(\frac{\partial u}{\partial x}+\frac{\partial v}{\partial y}+\varepsilon p\right) q d x d y=0 \\
\iint_{\Omega}\left[(R e-\alpha t) \frac{\partial u}{\partial t} w_{1}-\alpha x \frac{\partial u}{\partial x} w_{1}-\alpha y \frac{\partial u}{\partial y} w_{1}+R e\left(u \frac{\partial u}{\partial x} w_{1}+v \frac{\partial u}{\partial y} w_{1}\right)\right. \\
\left.-p \frac{\partial w_{1}}{\partial x}+(1+K)\left(\frac{\partial u}{\partial x} \frac{\partial w_{1}}{\partial x}+\frac{\partial u}{\partial y} \frac{\partial w_{1}}{\partial y}-K \frac{\partial N}{\partial y} w_{1}\right)\right] d x d y \\
\quad+\int_{\Gamma_{2}} p w_{1} d s+(1+K) \int_{\Gamma_{2}} w_{1} \frac{\partial v}{\partial y} d s=0 \\
\iint_{\Omega}\left[(R e-\alpha t) \frac{\partial v}{\partial t} w_{2}-\alpha x \frac{\partial v}{\partial x} w_{2}-\alpha y \frac{\partial v}{\partial y} w_{2}+\operatorname{Re}\left(u \frac{\partial v}{\partial x} w_{2}+v \frac{\partial v}{\partial y} w_{2}\right)\right. \\
\left.\quad-p \frac{\partial w_{2}}{\partial y}+(1+K)\left(\frac{\partial v}{\partial x} \frac{\partial w_{2}}{\partial x}+\frac{\partial v}{\partial y} \frac{\partial w_{2}}{\partial y}\right)+K \frac{\partial N}{\partial x} w_{2}\right] d x d y=0 \\
\left.\quad+K\left(2 N+\frac{\partial u}{\partial y}-\frac{\partial v}{\partial x}\right) w_{3}+\left(1+\frac{K}{2}\right)\left(\frac{\partial N}{\partial x} \frac{\partial w_{3}}{\partial x}+\frac{\partial N}{\partial y} \frac{\partial w_{3}}{\partial y}\right)\right] d x d y \\
\quad-\left(1+\frac{K}{2}\right) \int_{\Gamma_{2}} w_{3} N / x d s=0 .
\end{gathered}
$$




\section{Fully discretized iterative finite element scheme}

Similar to the approaches discussed in [16] and [29], in this paper, solutions of the weak formulation (3.2a)-(3.2c) and (3.3a)-(3.3d) are approximated by a finite difference scheme in time and a conformal $C^{0}$ finite element method in space. In the temporal direction, explicit-implicit(or semi-implicit) first order schemes are often adopted, namely the nonlinear terms are approximated explicitly and the linear terms are treated implicitly.

We suppose that $V_{2}^{h} \subset H^{1}(\Omega)$ is the finite element space for velocity $u, v$ and the space for pressure $p$ is $V_{1}^{h} \subset L^{2}(\Omega)$. For the micropolar fluid model, finite element space for micro-rotation $N$ is also $V_{2}^{h} \subset H^{1}(\Omega)$. As usual, we shall take $V_{2}^{h}$ to be piecewise quadratic polynomials $P_{2}$ and $V_{1}^{h}$ to be linear polynomials $P_{1}$, which is the standard Taylor-Hood finite element for the velocity-pressure pair, satisfying the inf-sup condition. If $\Delta t>0$ represents a time step size, and $\left(u_{h}^{n}, v_{h}^{n}, p_{h}^{n}\right)$ is an approximation of

$$
u\left(t^{n}\right)=u(n \triangle t), \quad v\left(t^{n}\right)=v(n \triangle t), \quad p\left(t^{n}\right)=p(n \triangle t)
$$

respectively, at the time $t=n \triangle t$. Then the approximation solution $\left(u_{h}^{(n+1)}, v_{h}^{(n+1)}, p_{h}^{(n+1)}\right)$ in $\left(V_{2}^{h}\right)^{2} \times V_{1}^{h}$ at the next time $t=(n+1) \Delta t$ can be obtained by the following typical temporal iterative scheme,

$$
\begin{gathered}
\iint_{\Omega}\left(\frac{\partial u_{h}^{(n+1)}}{\partial x}+\frac{\partial v_{h}^{(n+1)}}{\partial y}+\varepsilon p_{h}^{(n+1)}\right) q d x d y=0, \\
\iint_{\Omega}\left[(R e-\alpha t) \frac{u_{h}^{(n+1)}-u_{h}^{n}}{\triangle t} w_{1}-\alpha x \frac{\partial u_{h}^{n}}{\partial x} w_{1}-\alpha y \frac{\partial u_{h}^{n}}{\partial y} w_{1}+\operatorname{Re}\left(u_{h}^{n} \frac{\partial u_{h}^{n}}{\partial x} w_{1}+v_{h}^{n} \frac{\partial u_{h}^{n}}{\partial y} w_{1}\right)\right. \\
\left.\quad-p_{h}^{(n+1)} \frac{\partial w_{1}}{\partial x}+\left(\frac{\partial u_{h}^{(n+1)}}{\partial x} \frac{\partial w_{1}}{\partial x}+\frac{\partial u_{h}^{(n+1)}}{\partial y} \frac{\partial w_{1}}{\partial y}\right)\right] d x d y \\
\quad+\int_{\Gamma_{2}} p_{h}^{(n+1)} w_{1} d s+\int_{\Gamma_{2}} w_{1} \frac{\partial v_{h}^{(n+1)}}{\partial y} d s=0, \\
\iint_{\Omega}\left[(\operatorname{Re}-\alpha t) \frac{v_{h}^{(n+1)}-v_{h}^{n}}{\triangle t} w_{2}-\alpha x \frac{\partial v_{h}^{n}}{\partial x} w_{2}-\alpha y \frac{\partial v_{h}^{n}}{\partial y} w_{2}+\operatorname{Re}\left(u_{h}^{n} \frac{\partial v_{h}^{n}}{\partial x} w_{2}+v_{h}^{n} \frac{\partial v_{h}^{n}}{\partial y} w_{2}\right)\right. \\
\left.-p_{h}^{(n+1)} \frac{\partial w_{2}}{\partial y}+\left(\frac{\partial v_{h}^{(n+1)}}{\partial x} \frac{\partial w_{2}}{\partial x}+\frac{\partial v_{h}^{(n+1)}}{\partial y} \frac{\partial w_{2}}{\partial y}\right)\right] d x d y=0,
\end{gathered}
$$

for all $\left(w_{1}, w_{2}, q\right) \in\left(V_{2}^{h}\right)^{2} \times V_{1}^{h}$.

For the micropolar fluid model $u, v, N \in V_{2}^{h}, p \in V_{1}^{h}$, then by the similar pattern, the approximation solution $\left(u_{h}^{(n+1)}, v_{h}^{(n+1)}, N_{h}^{(n+1)}, p_{h}^{(n+1)}\right)$ in $\left(V_{2}^{h}\right)^{3} \times V_{1}^{h}$ at the time $t=$ 
$(n+1) \triangle t$ can be obtained by the following iterative finite element scheme,

$$
\begin{aligned}
& \iint_{\Omega}\left(\frac{\partial u_{h}^{(n+1)}}{\partial x}+\frac{\partial v_{h}^{(n+1)}}{\partial y}+\varepsilon p_{h}^{(n+1)}\right) q d x d y=0, \\
& \iint_{\Omega}\left[(R e-\alpha t) \frac{u_{h}^{(n+1)}-u_{h}^{n}}{\Delta t} w_{1}-\alpha x \frac{\partial u_{h}^{n}}{\partial x} w_{1}-\alpha y \frac{\partial u_{h}^{n}}{\partial y} w_{1}+R e\left(u_{h}^{n} \frac{\partial u_{h}^{n}}{\partial x} w_{1}+v_{h}^{n} \frac{\partial u_{h}^{n}}{\partial y} w_{1}\right)\right. \\
& \left.-p_{h}^{(n+1)} \frac{\partial w_{1}}{\partial x}-K \frac{\partial N_{h}^{n}}{\partial y} w_{1}+(1+K)\left(\frac{\partial u_{h}^{(n+1)}}{\partial x} \frac{\partial w_{1}}{\partial x}+\frac{\partial u_{h}^{(n+1)}}{\partial y} \frac{\partial w_{1}}{\partial y}\right)\right] d x d y \\
& +\int_{\Gamma_{2}} p_{h}^{(n+1)} w_{1} d s+(1+K) \int_{\Gamma_{2}} \frac{\partial v_{h}^{(n+1)}}{\partial y} w_{1} d s=0 \\
& \iint_{\Omega}\left[(R e-\alpha t) \frac{v_{h}^{(n+1)}-v_{h}^{n}}{\Delta t} w_{2}-\alpha x \frac{\partial v_{h}^{n}}{\partial x} w_{2}-\alpha y \frac{\partial v_{h}^{n}}{\partial y} w_{2}\right. \\
& +R e\left(u_{h}^{n} \frac{\partial v_{h}^{n}}{\partial x} w_{2}+v_{h}^{n} \frac{\partial v_{h}^{n}}{\partial y} w_{2}\right)-p_{h}^{(n+1)} \frac{\partial w_{2}}{\partial y}+K \frac{\partial N_{h}^{n}}{\partial x} w_{2} \\
& \left.+(1+K)\left(\frac{\partial v_{h}^{(n+1)}}{\partial x} \frac{\partial w_{2}}{\partial x}+\frac{\partial v_{h}^{(n+1)}}{\partial y} \frac{\partial w_{2}}{\partial y}\right)\right] d x d y=0 \\
& \iint_{\Omega}\left[(R e-\alpha t) \frac{N_{h}^{(n+1)}-N_{h}^{n}}{\triangle t} w_{3}-\alpha N_{h}^{(n+1)} w_{3}-\alpha x \frac{\partial N_{h}^{n}}{\partial x} w_{3}-\alpha y \frac{\partial N_{h}^{n}}{\partial y} w_{3}\right. \\
& +R e\left(u_{h}^{n} \frac{\partial N_{h}^{n}}{\partial x} w_{3}+v_{h}^{n} \frac{\partial N_{h}^{n}}{\partial y} w_{3}\right)+K\left(N_{h}^{(n+1)} w_{3}+\frac{\partial u_{h}^{n}}{\partial y} w_{3}-\frac{\partial v_{h}^{n}}{\partial x} w_{3}\right) \\
& \left.+\left(1+\frac{K}{2}\right)\left(\frac{\partial N_{h}^{(n+1)}}{\partial x} \frac{\partial w_{3}}{\partial x}+\frac{\partial N_{h}^{(n+1)}}{\partial y} \frac{\partial w_{3}}{\partial y}\right)\right] d x d y \\
& -\int_{\Gamma_{2}}\left(1+\frac{K}{2}\right) N_{h}^{(n+1)} w_{3} d s=0
\end{aligned}
$$

for all $\left(w_{1}, w_{2}, w_{3}, q\right) \in\left(V_{2}^{h}\right)^{3} \times V_{1}^{h}$.

\section{Numerical results}

In this section, we present a numerical example. The computational domain $\Omega$ is $[x, y] \in[0,5] \times[-1,1]$ with a $40 \times 10$ grid shown in Fig. 2 for solving the cases when the channel is driven for injection or small suction. For large suctions, we should use finer meshes since boundary layers appearing at the two opposing walls. All the solutions are shown at $x=2$ and we choose $d t=0.0001$. The numerical results are obtained by using Freefem ++ platform, and MATLAB. 


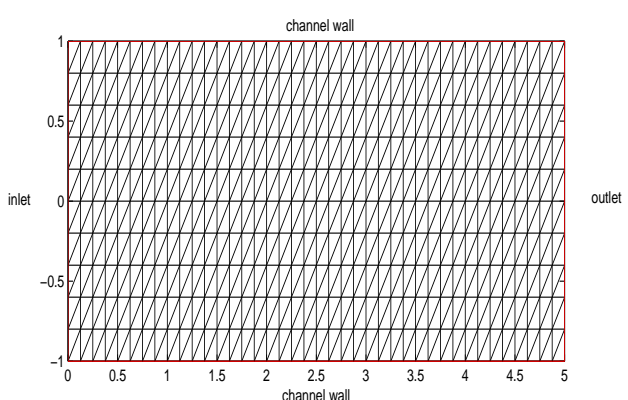

Figure 2: A triangular mesh of the rectangular channel domain $\Omega$.

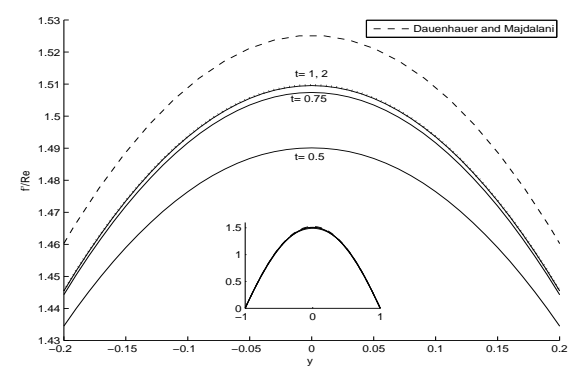

Figure 3: The time evolution of the axial velocity $F^{\prime} / R e$ for $R e=5, \alpha=0$.

\subsection{Numerical solutions for Newtonian fluid model}

In this part, cases of injection and suction are taken into consideration respectively.

we first consider two injection cases, $R e=5, \alpha=0$ and $R e=20, \alpha=-5$ and obtain a unique solution for each case which agrees substantially well with numerical solutions in [6]. The time evolution of the axial velocity for each solution at $t=0.5, t=0.75, t=$ $1, t=2$ is displayed in Figs. 3 and 4 respectively, from which we can have that the results beyond $t=1$ can not be visually distinguished, indicating that the steady-state solution obtained in [6] of each state may be stable.

Figs. 5-6 shows two solutions obtained for $R e=-20, \alpha=1$, a case of suction, where we can see that the two solutions match well with the analytical solutions in [14] at $t=0.01$. The figures also display the spatial variation of two different solutions with time. what's more, in order to make a clear view of the form of the solution at different time, we enlarge the images partly. However, the profile of every solution changes into different forms by time, thus an obvious conclusion can be drawn that the two steady-state solutions given in [14] may be unstable.

\subsection{Numerical solutions for micropolar fluid model}

We use the similar computation to study multiple solutions and their spatial variation for micropolar fluid model, a work has not been done by others. Thus in order to test the accuracy of our results, on the other hand, we obtain the approximation solutions 


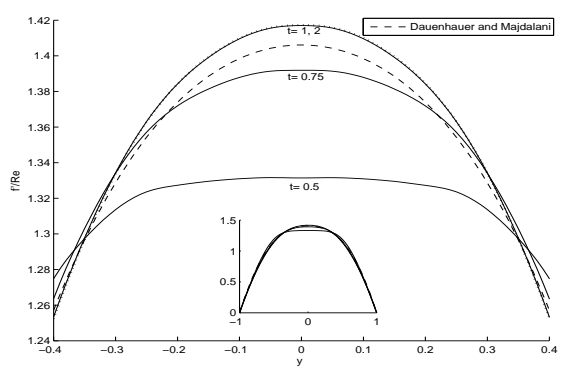

Figure 4: The time evolution of the axial velocity $F^{\prime} / R e$ for $R e=20, \alpha=-5$.

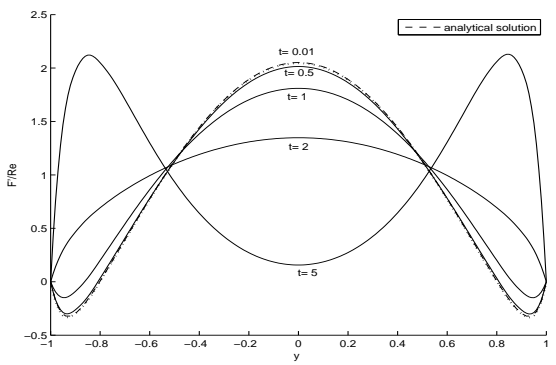

Figure 5: Evolution of $F^{\prime} / R e$ with time for the first solution under $R e=-20, \alpha=1$.

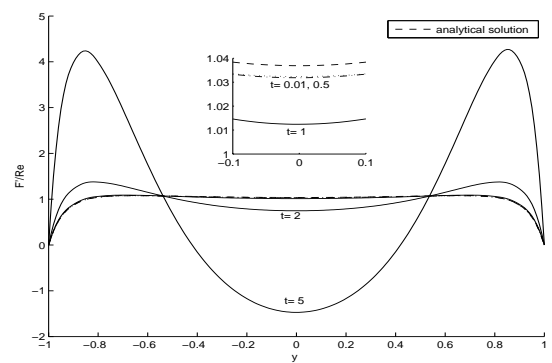

Figure 6: Evolution of $F^{\prime} / R e$ with time for the second solution under $R e=-20, \alpha=1$.

from the perspective of ordinary differential equations (15)-(16) in [24] with the help of the MATLAB boundary value problem solver bvp4c by following the footsteps of previous work. In our computation, we let $K=0.2$ and two different boundary parameters $s=0$ or $s=0.02$.

First, we investigate cases when the boundary parameter $s=0$, which means that the micro-elements close to the two walls of the channel are unable to rotate.

As Fig. 7 indicated, we obtain the steady-state solution given in [24] for $R e=5, \alpha=$ -3 , a case of injection, and we also can conclude that the unique solution given in [24] has reached the steady state, for at the scale used in the following image, the curves beyond $t=1$ are hardly to distinguish.

For suction driven channel, we investigate two cases when $R e=-9, \alpha=2$ and $R e=$ 

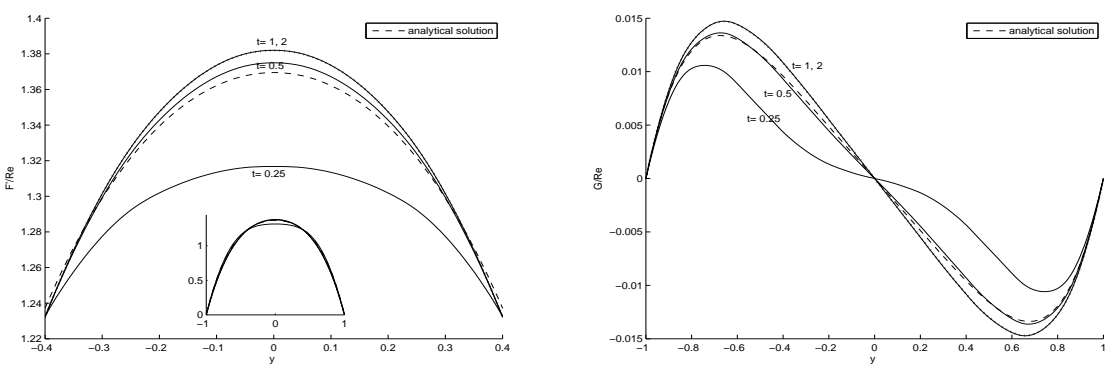

Figure 7: Spatial variation of $F^{\prime} / R e$ and $G / R e$ for $R e=5, \alpha=-3$ at several temporal time.
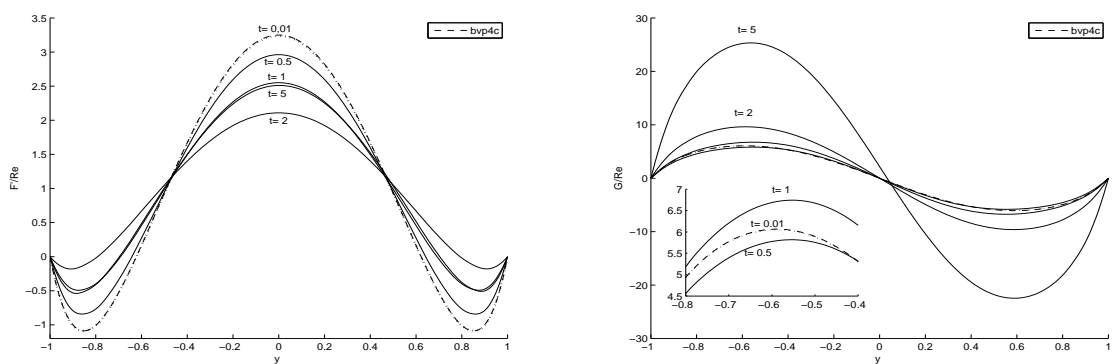

Figure 8: The time evolution of $F^{\prime} / R e$ and $G / R e$ for the first solution under $R e=-9, \alpha=2$.
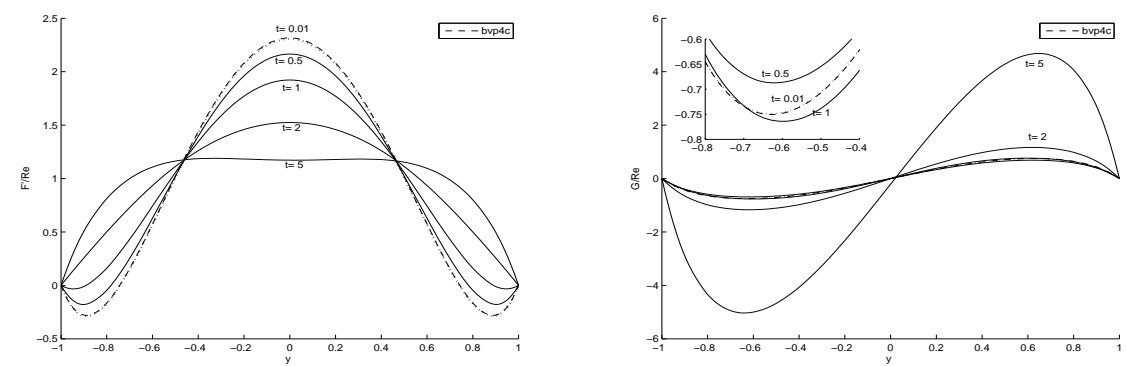

Figure 9: The time evolution of $F^{\prime} / R e$ and $G / R e$ for the second solution under $\operatorname{Re}=-9, \alpha=2$.

$-20, \alpha=1$. As for $R e=-9, \alpha=2$, two solutions are obtained by using finite element method, while three solutions are obtained when $R e=-20, \alpha=1$. Excellent agreement between our solutions and the solutions obtained through bvp4c is obvious at $t=0.01$. As is shown in Figs. 8-9 and Figs. 10-12, the profiles of these solutions vary with time and can not reach fixed forms respectively, which leads us to conclude that the steady state solutions obtained by bvp4c may be unstable.

Next, cases for $s=0.02$ are considered, meaning that the micro-elements near the walls are rotating, thus the values of $G / R e$ are not zero at the two walls. Similar behaviors can be observed as $s=0$ for injection and suction cases respectively. 

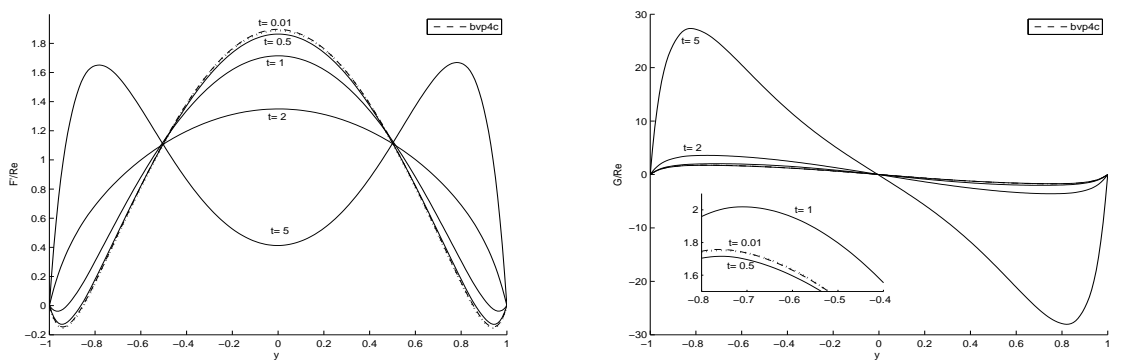

Figure 10: The time evolution of $F^{\prime} / R e$ and $G / R e$ for the first solution under $\operatorname{Re}=-20, \alpha=1$.
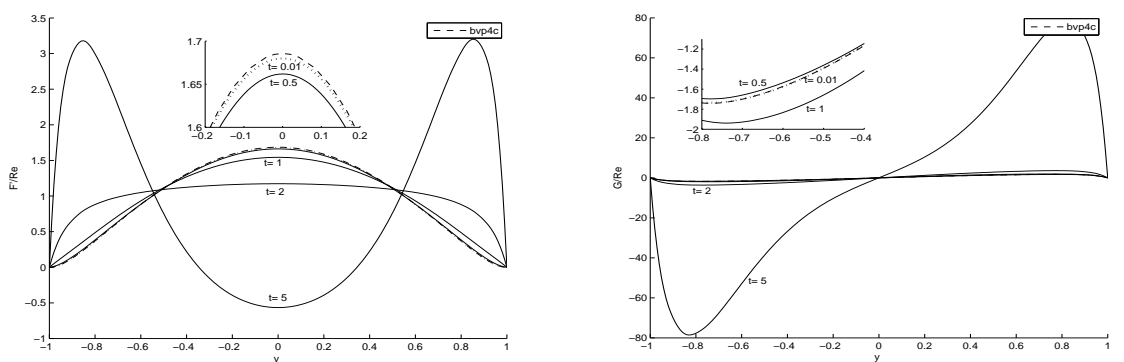

Figure 11: The time evolution of $F^{\prime} / R e$ and $G / R e$ for the second solution under $R e=-20, \alpha=1$.
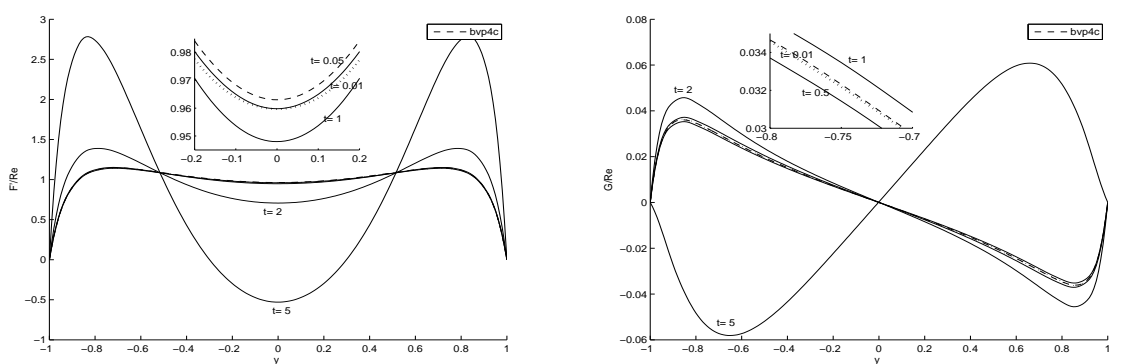

Figure 12: The time evolution of $F^{\prime} / R e$ and $G / R e$ for the third solution under $R e=-20, \alpha=1$.

Fig. 13 presents the time evolution of the unique solution obtained for $\operatorname{Re}=5, \alpha=1$, the results beyond $t=1$ become indiscernible, so the unique solution given in [24] is a stable solution.

As for $R e=-3, \alpha=-6$, a case of small suction, we can easily find the approximation analytical solution obtained in [24] by our direct computational method. From Fig. 14, a conclusion can be drawn that the steady-state solution given in [24] may be stable. Figs. 15-17 illustrate the profiles of three solutions obtained in the case of $R e=-30, \alpha=$ 1.5 and their spatial variation with time, Just as suction cases before, these steady-state solutions given by bvp4c may be unstable. In addition, the velocity vector field of the three 

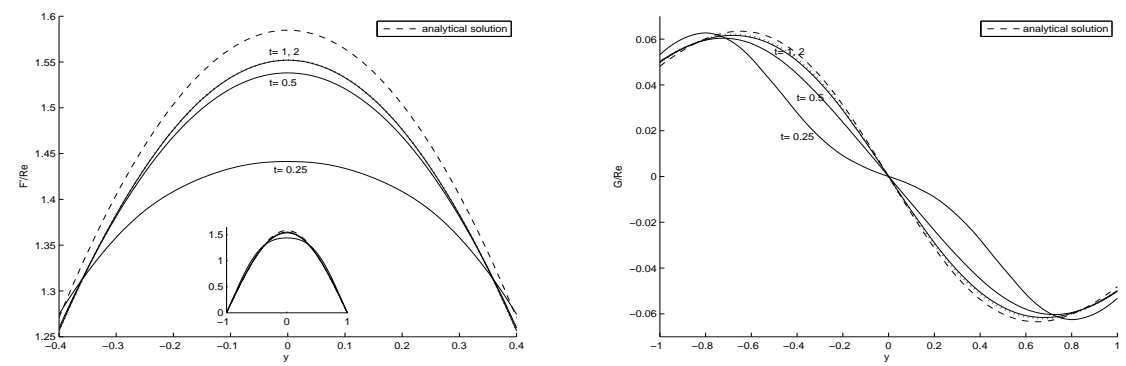

Figure 13: The time evolution of $F^{\prime} / R e$ and $G / \operatorname{Re}$ for $\operatorname{Re}=5, \alpha=1$.
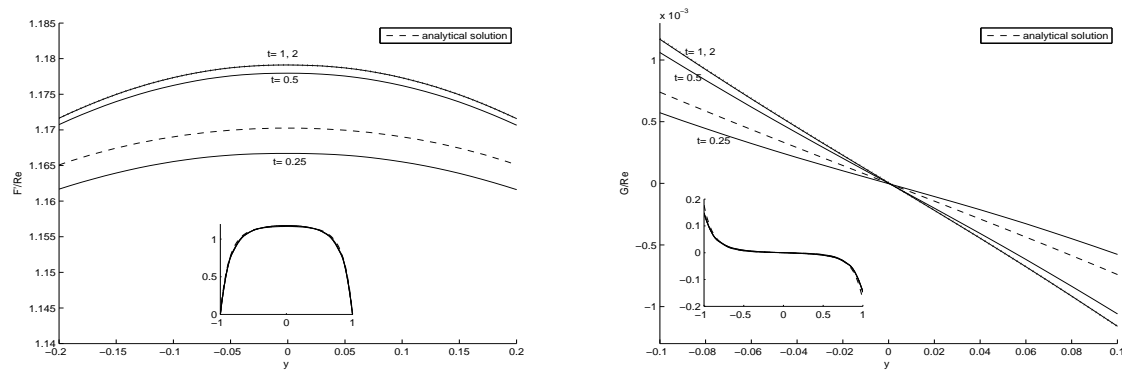

Figure 14: The time evolution of $F^{\prime} / R e$ and $G / R e$ for $R e=-3, \alpha=-6$.
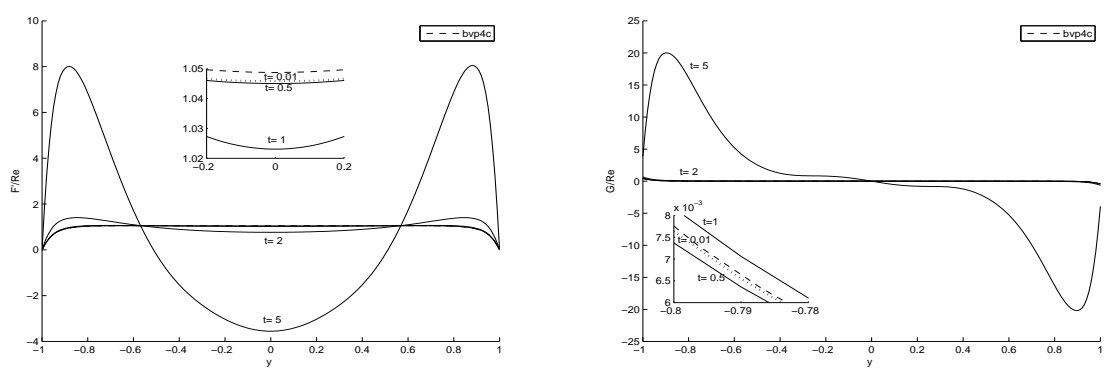

Figure 15: The time evolution of $F^{\prime} / R e$ and $G / R e$ for the first solution under $R e=-30, \alpha=1.5$.

solutions at $t=0.01$ in the whole channel are presented in Fig. 18 .

\section{Conclusions}

In this research, an explicit-implicit finite difference scheme in time and a continuous finite element method in space have been used to study multiple solutions for the laminar flow in a porous channel with expanding or contracting walls. Two models are considered, that is Newtonian fluid model and the micropolar fluid model. We first non-dimensionalize the governing equations of the two models and introduce the expansion ratio $\alpha$. Then we 

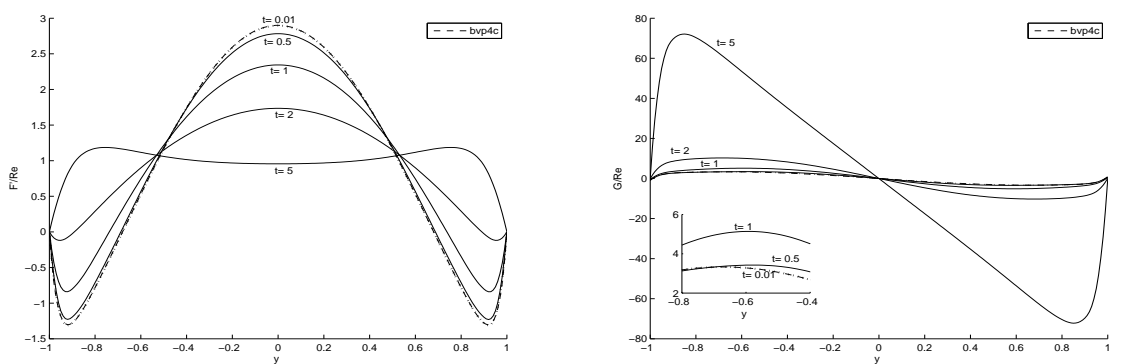

Figure 16: The time evolution of $F^{\prime} / R e$ and $G / R e$ for the second solution under $\operatorname{Re}=-30, \alpha=1.5$.
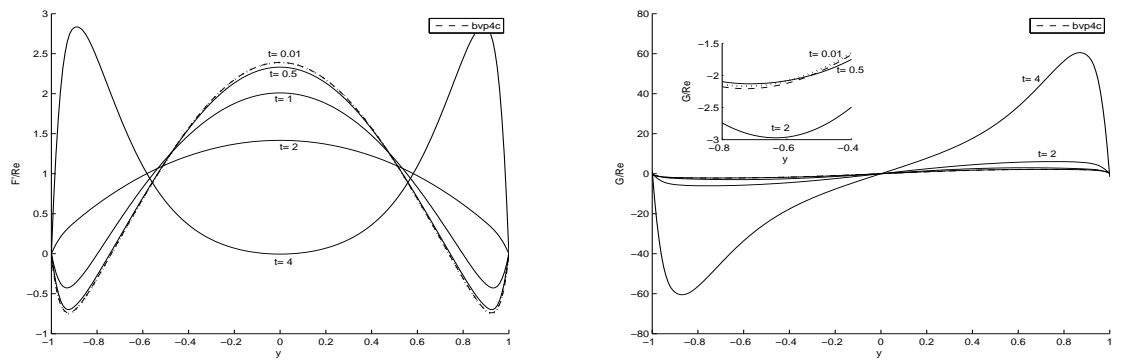

Figure 17: The time evolution of $F^{\prime} / R e$ and $G / R e$ for the third solution under $R e=-30, \alpha=1.5$.
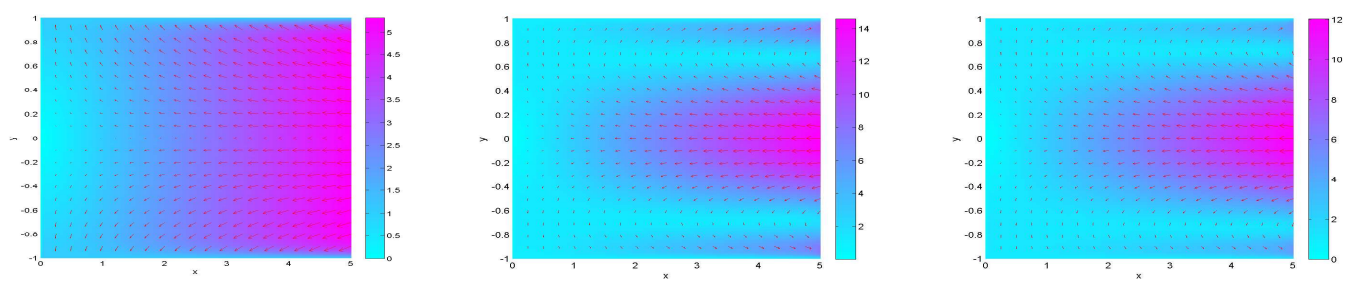

Figure 18: Plot of the velocity vector field of the first, second and third solution for $R e=-30, \alpha=1.5$ at $t=0.01$, respectively.

describe their weak formulations and the iterative finite element schemes, respectively. Some numerical solutions are obtained, and the main findings of this study can be summarized as follows: For the Newtonian fluid model, there is a unique solution which may be stable for the injection, while for large suctions, multiple solutions exist and the profile of each solution varies with time, which indicates that these solutions may be unstable; For micropolar fluid model, similar conclusions can be drawn as the Newtonian fluid model.

Acknowledgments This work is partially supported by the National Natural Science Foundations of China (No. 91430106) and the Fundamental Research Funds for the Central Universities (No. 06500073). 


\section{References}

[1] A. S. Berman, Laminar flow in channels with porous walls, J. Appl. Phys., 24 (1953), pp. 12321235.

[2] R. M. TERRILL, Laminar flow in a uniformly porous channel, Aeronautical Journal, 15 (1964), pp. 299-310.

[3] I. Proudman, An example of steady laminar flow at large Reynold number, J. Fluid Mech., 9(4) (2006), pp. 593-602.

[4] S. W. YuAN, Further investigation of laminar flow in channels with porous walls, J. Appl. Phys., 27 (1956), pp. 267-269.

[5] M. Morduchow, On laminar flow through a channel or tube with injection: application of method of averages, J. Appl. Math., 14 (1957), pp. 361-368.

[6] E. C. Dauenhauer AND J. MAJdALANi, Exact self-similarity solution of the Navier-Stokes equations for a porous channel with orthogonally moving walls, Phys. Fluids, 15 (2003), pp. 14851495.

[7] G. Raithby, Laminar heat transfer in the thermal entrance region of circular tubes and twodimensional rectangular ducts with wall suction and injection, Int. J. Heat Mass Tran., 14 (1971), pp. 223-243.

[8] W. A. RoBinson, The existence of multiple solutions for the laminar flow in a uniformly porous channel with suction at both walls, J. Eng. Math., 10 (1976), pp. 23-40.

[9] J. Sobey and G. Drazin, Bifurcations of two-dimensional channel flows, J. Fluid Mech., 171 (1986), pp. 263-287.

[10] M. B. Zaturska, P. G. Drazin And W. H. H. Banks, On the flow of a viscous fluid driven along a channel by suction at porous walls, Fluid Dyn. Res., 4 (1988), pp. 151-178.

[11] E. B. B. Watson, W. H. H. Banks, M. B. Zaturska and P. G. Drazin, On transition to chaos in two-dimensional channel flow symmetrically driven by accelerating walls, J. Fluid Mech., 212 (1990), pp. 451-485.

[12] L. DurLofsky AND J. F. BRADY, The spatial stability of a class of similarity solutions, Phys. Fluids, 27 (1984), pp. 1068-1076.

[13] S. M. CoX AND A. C. KING, On the asymptotic solution of a high-order nonlinear ordinary differential equation, Proc. R. Soc. London, Ser. A, 453 (1997), pp. 711-728.

[14] H. Xu, Z. L. Lin, S. J. LiaO, J. Z. Wu AND J. Majdalani, Homotopy based solutions of the NavierStokes equations for a porous channel with orthogonally moving walls, Phys. Fluids, 22 (2010), pp. 1-18.

[15] X. H. Si, L. C. Zheng, X. X. Zhang And Y. Chao, Existence of multiple solutions for the laminar flow in a porous channel with suction at both slowly expanding and contracting walls, International Journal of Minerals, Metallurgy and Materials, 18 (2011), pp. 494-501.

[16] H. X. XU, P. LiN AND X. H. SI, A study of multiplt solution for the Navier-Stokes equations by a finite element method, Numer. Math. Theor. Meth. Appl., 7 (2014), pp. 107-122.

[17] A. C. ERIngen, Theory of micropolar fluids, Indiana University Mathematics Journal, 16(1) (1965): 42.

[18] A. C. ERIngen, Theory of themomicropolar fluids, J. Math. Anal. Appl., 38(2) (1972), pp. 480496.

[19] M. T. Darvishi, F. Khani, F. G. Awad, A. A. Khidir and P. Sibanda, Numercial investigation of the flow of a micropolar fluid through a porous channel with expanding or contracting walls, Propulsion and power Reserach, 3 (2014), pp. 133-142.

[20] M. Ashraf, M. Anwar Kamala And K. S. Syeda, Numercial study of asymmetric laminar flow of micropolar fluids in a porous channel, Comput. Fluids, 38 (2009), pp. 1895-1902. 
[21] M. M. Rashidi, S. A. Monhimanian Pour And N. Laraqi, A semi-analytical solution of micropolar flow in a porous channel with mass injection bu using differential transfer method, Nonlinear Anal-Model, 15(2010), pp. 341-350.

[22] Z. ZiabakHSh AND G. DomairRY, Homotopy analysis solution of micropolar flow in a porous channel with high mass transfer, Adv. Theor. Appl. Mech., 1 (2008), pp. 79-94.

[23] A. A. Joneidi, D. D. Ganji And M. Babaelahi, Micropolar flow in a porous channel with high mass transfer, Int. Commun. Heat Mass, 36 (2009), pp. 1082-1088.

[24] X. H. Si, L. C. Zheng AND P. Lin, Flow and heat transfer of a micrpolar fluid in a porous channel with expanding or contracting walls, Int. J. Heat Mass Tran., 67 (2013), pp. 885-895.

[25] P. LiN, A sequential regularization method for time-dependent incompressible Navier-Stokes, SIAM. Numer. Anal., 34 (1997), pp. 1051-1071.

[26] P. Lin, X. Q. Chen And M. T. Ong, Finite element methods based on a new formulation for the non-stationary incompressible Navier-Stokes equations, Int. Numer. Meth. Fluids, 46 (2004), pp. 1169-1180.

[27] P. Lin AND C. Liu, Simulations of singularity dynamics in liquid crystal flows: A finite element approach, J. Comput. Phys., 215 (2006), pp. 348-362.

[28] J. HuA, P. Lin, C. Liu AND Q. WANG, Energy law preserving $C_{0}$ finite element schemes for phase field models in two-phase flow computations, J. Comput. Phys., 230 (2011), pp. 7115-7131.

[29] H. D. SHI, P. Lin, B. T. Li AND L. C. ZHENG, A finite element method for the heat transfer of powerlaw flow in channels with a transverse magnetic field, Math. Method. Appl. Sci., 37 (2014), pp. 1121-1129. 